\title{
Electromagnetic radiation originating from unstable electron oscillations
}

\author{
Juul Rasmussen, J.; Pécseli, Hans
}

Publication date:

1976

Document Version

Publisher's PDF, also known as Version of record

Link back to DTU Orbit

Citation (APA):

Juul Rasmussen, J., \& Pécseli, H. (1976). Electromagnetic radiation originating from unstable electron oscillations. Risø National Laboratory. Risø-M No. 1818

\section{General rights}

Copyright and moral rights for the publications made accessible in the public portal are retained by the authors and/or other copyright owners and it is a condition of accessing publications that users recognise and abide by the legal requirements associated with these rights.

- Users may download and print one copy of any publication from the public portal for the purpose of private study or research.

- You may not further distribute the material or use it for any profit-making activity or commercial gain

- You may freely distribute the URL identifying the publication in the public portal

If you believe that this document breaches copyright please contact us providing details, and we will remove access to the work immediately and investigate your claim 


\section{Title and author(s)}

Electromagnetic radiation originating from unstable electron oscillations

by

J. Juul Rasmussen and H.L. Pêcseli
Date

April 1976

Department or group

Physics

Group's awn registration number(s)

Abstract

$$
13 \text { pages }+ \text { tables }+14 \text { illustrations }
$$

Electronagnetic radiation in the range $200-700 \mathrm{MHz}$ was observed from an unmagnetized argon discharge. The radiation is attributed to standing surface waves spontaneously excited due to an unstable electron distribution. It is argued that, due to the finite length of the system, the electromagnetic radiation can be considered as if originating from a finite number of dipole antennas orientated along the discharge axis. 
ISBN $87 \quad 550 \quad 03559$ 


\section{Introduction}

An experiment was designed in order to investigate possirle electromagnetic radiation arising from electrostatic electron oscillations in a plasma. Various theoretical works on this sulject are presented in the literature, some concentrated upon a discussion of the electromagnetic radiation connected with the type II and III bursts in the solar corona. see, for example, refs. $1-6$.

These theories ecrentially explain the coupling at a plasma boundary between a longitudinal plasma oscillation and an electromagnetic wave by assuming that the wave vector has a slight angle with respect to the vector $\nabla n$ giving rise to an oscillating dipole layer along the (plane) plasma koundary. This dipole layer has a wave-length parallel to the boundary and moves with a phase velocity larger than or equal to the light velocity, $c$, thus appearing as a fast surface wave that couples to propagating electromagnetic waves outside the plasma. In the solar corona, longitudinal plasma oscillations are believed to re excited by a two-stream instability arising in connection with solar bursts, giving $r i$ se to electromagnetic waves in the $\mathrm{mHz}$ range received on the earth. Experiments that demonstrate this coupling between longitudinal plasina oscillations and transverse electromagnetic waves have been reported (e.g. ref. 7 ). In the following we present results from an experiment in an unmagnetized argon discharge where electron oscillations are spontaneously excited due to an unstable, two-stream type, electron velocity distribution function. Due to reflections at the ends of the column, a standing wave is obtained. The wave has the nature of a slow surface wave. We interpret the observed electromagnetic radiation as resulting from the incomplete compensation of the displacement currents by the Maxwell term in a finite plasma or, in other words, resulting from electric fields outside the plasma connecting regions of positive and negative charge density of the standing wave. Seen from the far field, we may conslder a standing wave of $\mathrm{N}$ wavelengths as an array of $\mathrm{N}$ oscillating dipoles orientated in the direction of the wave vector. Although glov: surface waves are "non-radiating", 


$$
-2-
$$

a standing wave with a finite number of wavelengths, $N$, will radiate with the radiation pattern determined by elementary principles for radiation from $N$ oscillating dipoles. 
II. Experimental Set-Up

The experiment was carried out in an argon discharge shown schematically on fig. 1. The plasma is created by lonization of the neutral gas by fast electrons emitted from the negatively biased cathode and accelerated towards the grounded grid. The cathode consists of closely wound tungsten filaments $(00.2 \mathrm{~mm}$. heated to $22000 \mathrm{~K}$ by a DC-current. The cathode bias was variable from 0 to $-200 \mathrm{~V}$. The grid was made of $0.05 \mathrm{~mm}$ nickel wires with $0.5 \mathrm{~mm}$ spacing. The plasma was terminated by a movable conducting end-plate with a maximum distance from the grid of $25 \mathrm{~cm}$. A pyrex-glass tube (inner diameter $210 \mathrm{~cm}$. ) with wall thickness $5 \mathrm{~mm}$ surrounded the discharge. The argon neutral pressure was in the region of 0.5-20 mtorr.

Figure 2 shows a current-voltage characteristic of the discharge. As expected, the current, $I$, increases rapidly with cathode potential, for $\phi<-16 \mathrm{~V}$ corresponding to the ionization potential $15.76 \mathrm{eV}$ for argon.

The electron velocity distribution at a position between the grid and the end-plate consists of two components: fast electrons from the cathode that have not yet collided and a group of "thermal" electrons produced by the ionization. The two shielded Langmuir probes, shown on fig. 1, allow investigations of both components. The probes are made of tungsten wires with a diameter of $0.2 \mathrm{~mm}$ and $5 \mathrm{~mm}$ in length. The shields are made of stainless steel. Our interpretation of the probe measurements are based on the assumption that the probe facing towards the cathode (probe 1) collects electrons both from the beam and the backgrounc, while the probe facing the opposite direction (probe 2) has access to the background ("thermal") electrons only. Probe 2 therefore exhibits the characteristics of a Langmuir probe in a temperate plasma. The characteristics of probe 1 , for probe biases larger (less negative) than the blas of the cathode, are similar to those of probe 2 but diaplaced in the direction towards pure electron current by an amount determined by the current (flux) of the electron beam, since the beam electrons have sufficient energy to reach the probe. Figure 3 shows such probe characteristics. For probe blases below (more negative than) the cathode bias, we should in principle be able to analyze the beam also. Unfortunately, the characteristics are 
rather distorted in this regior. (Figure 3 shows a slight bending of the lower trace due to the beam temperature.) In order to check whether these rather crude assumptions give reasonable results, we investigated the spatial variation of the beam $f l u x$ and the density and temperature of the background ("thermal") electron component at relatively high neutral pressures. Figures $4 \mathrm{a}$ and $4 \mathrm{~b}$ show measured beam fluxes and background densities (logarithmic scale) as a function of position for two different Ar-pressuzes. The background density, $n$, decreases away from the cathode with an e-folding length $215 \mathrm{~cm}$, roughly independent of Ar-pressure and cathode bias. The beam flux, on the other hand, shows a spatial decrease with an efolding length strongly dependent on neutral pressure. By assuming the collision probability of a beam electron with an argon atom in an interval $\mathrm{dx}$ to be $\mathrm{dx} / \mathrm{l}$, there $\mathrm{l}$ is the mean free path for collisions, we have the beam flux, assuming that an electron is lost from the beam after one collision, $n_{b} v_{b}=n_{o b} v_{o b} \exp (-x / l)$. Using the relation

$$
l=\frac{4 \kappa T_{A r}}{\pi P_{a r} D^{2}}
$$

where $\mathrm{T}_{\mathrm{Ar}}, \mathrm{P}_{\mathrm{Ar}}$ and $\mathrm{D}_{\mathrm{Ar}}$ are the neutral temperature, pressure and atomic diameter, respectively, we find $l=6 \mathrm{~cm}$ for $p_{A r} \approx 6$ mtorr and $\ell=12 \mathrm{~cm}$ for $p_{A r} \simeq 3$ mtorr. These $\ell$ vallies are in good agreement with the measured e-folding lengths for the flux variation. (We used a cross section for eleciron-argon collisions $\sigma=8 \cdot 10^{-16} \mathrm{~cm}^{2}$ according to ref. 81 . We may thus conclude that our interpretation of the Langmuir probe measurements cannot be entirely erroneous, which is probabiy the best conclusion attainabie with the present probe geometry. Figure 5 shows the radial variation of $n$ and $n_{b} v_{b}$ for two different positions. We can estimate the electron density of the beam by assuming that an electron is lost from the beam after one collision and that the beam velocity is constant dctermined by the difference between the cathode potential and the plasma potential. We find $n_{b} / n \sim 10^{-2}$ at a distance of $\sim 1 \mathrm{~cm}$ behind the qrid. At low neutral pressures this ratio was practically constant along the discharge. Knowirig the total current through the 
system, we find that only a few per cent of the electrons emitted from the cathode manage to get through the grid.

From probe characteristics such as those shown in fig. 3 we found the electron temperature of the background to be $\mathrm{T}_{\mathrm{e}}=3-4 \mathrm{eV}$ with a weak dependence on neutral pressure and cathode bias. For Ar-pressures below $\sim 10$ mtorr, we found $T_{e}$ independent of position. At higher pressures we observed an increase in $T_{e}$ as the probe was moved from the grid towards the end-plate, the variation being up to $\sim 20$ at a distance of $25 \mathrm{~cm}$. We have no explanation for this effect. The possihility of electron heating by the unstable oscillations was taken into account, but we saw no correlation between the temperature variation and the observed wave phenomena. The temperature variation itself is not improbable since the electron thermal conductivity is decreased by the neutrals at these high pressures. The beam temperatures, $T_{b}$, cannot be measured directly for the reasons already mentioned, but we may assume that it is determined by the potential variation across the cathode giving $\mathrm{T}_{\mathrm{b}} 22 \mathrm{~T}_{\mathrm{e}}$. In Fig. 6 we show the variation of the floating potential along the discharge measured on probe 2. At low neutral pressures we observe a potential decrease with distance from the grid in agreement with the density variation shown on Fig. $4 b$, if we assume that the electrons are Boltzmann distributed

$$
\phi e=K T_{e} \ln \left(n(x) / n_{0}\right)
$$

with $T_{e}$ constant. At higher neutral pressures, the floating potential tended to increase with distance. In these cases, however, we observed a variation of $T_{e}$ also, as already noted, so eq. (2) is invalid.

Finally Fig. 7 shows the density variation, as measured on probe 2 at a given position as a function of discharge current. We observe a roughly linear relation as expected for this and similar devices. 9 ) We feel, however, that the scatter in measured densities is too large to allow us to use the discharge current as a measure of plasma density, as several authors suggest. In the following all density measurements refer to Langmutr probe measurements (probe 2). 
A recent work by V.P. Pashchenko et a ${ }^{10}$ provides interesting discussions on voltage, current and density relations in simple discharges with a hot cathode and a cold anode. 


\section{Wave Measurements.}

Wave measurements in the plasma are performed by an ordinary Langmuir probe (not shown in Fig. 1). Outside the plasma elect-omagnetic waves are detected on half-wave dipole antenna at a distance of several wavelergths from the plasma column (i.e., outside the "near field"). The signal is analyzed on a Hewlett-Packard spectrum analyzer covering the range $100 \mathrm{kHz}$ $-1.3 \mathrm{GHz}$. (During the measurements in question the length of the plasma column was 5-10 cm and the density approximately constant in the axial direction).

The electromagnetic radiation outside the plasma always occur simultaneously with the oscillations in the plasma. Figure 8 shows typical spectra. When surrounding the discharge by a grounded metal cage, we observed a decrease in the amplitude of the electromagnetic oscillations outsic the cage by a factor of more than 250 , thus ensuring that the oscillations did originate from the plasma. (1) The electromagnetic oscillations were polarized parallel to the discharge axis, and the radiation pattern was, in all cases, investigated, roughly symmetric with respect to a plane perpendicular to the discharge axis. See Fig. 9 .

We interpret the electromagnetic radiation as follows: The electron beam has a velocity corresponding to at least $\sim 16 \mathrm{eV}$ (for smaller beam velocities no plasma is created at all, see Fig. 2). The beam velocity is thus much larger than the thermal velocity of both the background and beam. Thermal effects may thus be neglected for long wavelengths, and the electron distribution considered as unstable with respect to a two-stream electrostatic instability. (Electromagnetic instabilities having characteristic wavelengths $\sim_{c} / \omega_{p}$ can be excluded). A detailed calculation of the real part of the dispersion-relation and the growth rate must take into account not only the radial variation of density but also the finite length of the plasma.2) we may, however, obtain a rough estimate by assuming a cold plasma column with constant density and infinite length. Assuming quasi-stationarity, we get the real part of the dispersion relation

$$
\Omega^{2}(k)=\tilde{\mu}_{p}^{2} I_{m}^{\prime}(k a) K_{m}(k a) k a
$$


where $a$ is the radius of the colunn and $I_{m}$ and $K_{m}$ the modified Bessel and Bankel functions, respectively. Equation (3). plotted on Fig. 10, is obtained by neglecting the beam entirely, since the beam density is very small, the most unstable waves will be those corresponding to a wa-number where the dielectric function of the beam alone is zero, since this case gives the strongest modification of the electron-electron interaction, 1.e.. $\alpha(k)-k v_{b}=\omega_{p b}=0$ since $\omega_{p b}<\Omega$. Due to the smallness of the beam density, the real part of the dispersion relation $w 11$ still be given by eq. (3). More detailed calculations may be found in refs. 13-15. Also the glass vall (with dielectric constant $\epsilon_{g}=4.82$ (Pyrex)) should be taken into account in a complete calculation. It will give rise to a backward wave region (see, for instance, ref. 17). This effect may, however, he masked by the radial density variation. A dispersion relation calculated for an infinity long plasma column (as e.g. eq. (3)) will in general not be the same as for a segment of this column. Calculations taking into account the finite length of the plasma are outlined in ref. 16. Observations of unstable waves of similar type (Trivelpiece - mode ${ }^{9)}$ ) in a magnetized plasma are reported in ref. 18. (We would like to mention one interesting point in connection with discharges of the present type: due to the unavoidable anisotropy of the distribution function, the dielectric tensor $\underline{E}$ will be anisotropic also. If $\underline{E}$ is t.sotropic $(\underline{\underline{E}}=\varepsilon \underline{\underline{\underline{1}}})$, then $\nabla \cdot \varepsilon \cdot \underline{E}=\varepsilon \nabla \cdot \underline{E}=0$ o: $\nabla \cdot \underline{E}=0$, i.e., the electric field is divergence-free, since $\varepsilon=0$ is no solution for bounded plasma. For anisotropic $\underline{E}$ in general $\nabla \cdot \underline{E} \neq 0$, which may give the solutions entirely different features. In our case, however, this effect is of minor importance since the anisotropy is negligible because of the small beam density.)

Only waves travelling from the grid towards the end-plate will be unstable, but wave reflections at the steep density gradients at the end-plate and grid will give rise to a standing wave for rather a standing wave component) with, say, N wavelengths. The distribution of the electric field lines outside the plasma is sketched on Fig. 11. Seen from the far fleld, a standing wave of $N$ wavelengths may be viewed as an array of $N$ oscillating dipoles orlentated along the axis, and it will radiate accordingly 
(although slow surface waves with a dispersion relation given by eq. (3) are non-radiating). Dy carefully adjusting the movable end-plate and the discharge current, we can obtain radiation patterns with 2 or 3 main lokes corresponding to $N=2$ or $N=3$. See Fig. $9 a$. For higher $N$, the radiation pattern becomes somewhat irregular, Fig. 9b. (The assywnetry of the radiation patterrs is attributed to screening of unavoidable metal parts of the set-up rather than to assymetry of the plasma oscillations). issuming $m=0$ for the plasma oscillations. we explain the axiparallel polarization of the electromagnetic waves.

In Fig. 12 we show $\Omega / \sqrt{2}$ (in arbitrary units) as a function of $k=\Omega / V_{b}$ were $\Omega$ is the observed frequency on the antenna outside the plasma. This k-value will generally be larger than or equal to the $k$-value of the most unstable oscillations in the discharge since $k=\Omega / v_{b}$ need not satisfy the standing wave condition: $k=29 N / L$ where $L$ is defined on Fig. 1 . Since there are several wavelengths in the system $\Omega / V_{b}$ will be close to the true wavenumber of the most unstable oscillation and, assuming that we observe this one, we may interpret Fig. 12 as showing the dispersion relation of the oscillations. Since $2 \mathrm{TV}_{h} / \Omega$ gives fairly short wavelengths (smaller than $a$, the column radius). we expect $\Omega / \omega_{p} \sim \Omega / / n$ to be almost. independent of $k$ according to Fig. 10 in agreement with the observatiors. The more sophisticated calculations of refs. 14-15 do not alter this conclusion. The absolute magnitude of $\Omega$ agrees favourably with the predicted value calculated on the basis of the average density measured by the Langwuir probe.

The error bars on Fig. 12 indicate the band-width of the observed oscillations, while the circle shows the frequency with maximum anplitude. We attribute this line-width to the oscillations with smaller growth rates. It is well known that the growth rate for a two-stream instabllity in infinlte plasmas increases monotonically from $k_{m i n}=0$ to its maximum and then drops rapidly $\left.{ }^{20}\right)$ (in the experiment $k_{m i n}=2 \pi / L, 11 m i t e d$ by geometry). Referring to the dispersion relation shown on Fig. 10, we expect the frequency spectrum of the unstable oscillation to be strongly aesymetric with respect to the frequency with maximum amplitude. 
Figure 12 shows that this is indeed the case for at least a significant number of measurements. The waves with smaller growth rates may also explain some of the distortion of the radiation patterns.

Figure 13 show's the radial variation of the amplitude of the fluctuating potential in the plasma as measured by a simple Langmuir probe. The presence of the probe was observed to disturb the discharge somewhat, so Fig. 13 only gives a qualitative picture but the surface wave character of the plasma oscillations is clearly reflected by the radial increase in amplitude. The phase of the signal across the column was practically constant supporting the assumption of an $m=0$ mode. Some of the irregularities can, apart from being due to the presence of the probe, be attributed to excitation of other modes. sprradic bursts of radiation were in some cases observed to be very sensitive to neutral pressure and discharge current. This effect may be explained by standing waves between the grid and cathode. Our explanation of the radiation applies equally well to such waves. The coarse surface of the cathode (a tungsten spiral) makes it very difficult to satisfy the standing wave condition, thus explaining the sporadic nature of the oscillations. In a few cases second harmonic generation was observed. (See Fig. 14). This effect can only be explained by considering nonlinear effects beyond the scope of this report. 
IV. Conclusion

We have reported experimental observations of the coupling between electrostatic oscillations in a finite plasma and electromagnetic radiation outsiảe the plasma. Although our experiment may be of minor relevance to the explanation of, for instance, solar bursts, our interpretation suggests a simple mechanism for the coupling of electrostatic oscillations in a finite plasma to external electromagnetic radiation an explanation that may be applied to many experiments desiqned to simulate solar phenomena.

Another obvious application of the present radiation phenomena would be to construct an antenna for operation in the MHz-range. Unfortunately we found that the radiated power was very low, of the order of $1 \mu \mathrm{W}$. If, however, the power can be increased to an acceptable level a "plasma antenna", hased on our device, presents several advantages : low cost, easy frequency modulation (by modulating the cathode voltage), and a possibility of varying the radiation pattern. One might also construct a horn antenna by letting one side of the horn represent the endplate, while a hole covered with a fine meshed grid at the opposite side substitutes for the grid on Fig. 1. The cathode could be placed outside the horn. A vacuum system would not present any problems in the pressure range in question. We found the radiation to be very sensitive to mechanical vibration in the grid and the end-plate, and to variations in neutral pressure and cathode temperature. These problems are, however, easily solved. Also the band-width of the spectrum can be decreased by decreasing the beam temperature. This can be oktained by using a cathode with a smaller potential drop than in our case. Applications of "plasma antennas" have been suggested earlier (see, for instance, ref. 16 for a brief review), but always with the plasma playing a passive role : the waves were excited by some external means ${ }^{15,16)}$. In the present case the radiation originates from inherent oscillations in the plasma. Alternatively, there might be advantages in using the radiated spectrum for diagnostic purposes. Some of our results have already been published in ref. 21 . During the preparation of this manuscript our attention was 
drawn to a recently related work by Popovitch and Kharchenko 221 where HF-radiation due to electron oscillations in a beamplasma discharge was observed.

\section{Acknowledgements}

We thank M. Nielsen and B. Reher for their skillful technical assistance. 


\section{REFERENCES}

1) D. A. Tidman, Phys. Rev. 117,366 (1960).

2) D. A. Tidman and G. H. Weiss, Phys. Fluids 4, 703 (1961).

3) D. A. Tidman and J.M. Boyd, Phys. Fluids 5,213 (1962).

4) G. B. Field, Astrophys. J., 124, 555 (1956).

5) C. C. Harvey, Solar Phys., 40, 193 (1975).

6) D. F. Smith and W. D. Davis, Solar Dhys., 41, 439 (1975).

7) N. Ben-Yosef and A. S. Kaufman, Electronics Letters., 2., 176 (1966).

8) J. B. Hasted, Physics of Atomic Collisions., (2nd edition, London Butterworths, 1972), p. 307, 396.

9) A. W. Trivelpiece and R. W. Gould, J. Appl. Phys. 30, 1784, (1959).

10) V. P. Pashchenko, V. L. Mironovich, and I. P. Stakhanov, Sov. Phys. Tech. Phys., 19, 1332 (1975).

11) K. G. Emeleus and A. Garscadden, Naturwissenschaften, 47, 491 (1960).

12) W. H. Amein, V. V. Polgopolov, A. M. Hussein, and K. B. Zayed, Plasma Phys. 17, 497, (1975).

13) R. J. Briggs, in Advances in Plasma Physics Vol 4., ed.

A. Symon and $W$. B. Thompson (Intersience 1971) p. 43.

14) B. B. O'Brien, Plasma Phys. 9, 369 (1967).

15) 0 . Nyman, $A$ 'Sheoretical and Experimental Investigation of Wave Propagation in a Plasma Waveguide (Thesis, in Danish) Laboratory of Electromagnetic Theory, Lyngby, Denmark., Rep. N. LD9 (1966).

16) J. Bach Andersen, Metallic and Dialectric Antennas, (Polyteknisk Forlag 1971).

17) W. P. Allis, S. J. Buchsbaum, and A. Bers: waves in anisotropic plasmas (M.I.T. Press, 1963) P. 220.

18) T. Idehara, N. Miyama, and $X$. Ishida, Phys. Lett. $\underline{53}$, 97, (1975).

19) C. W. Roberson, A. S. Ratner, and J. L. Hirschfield, Phys. Rev. Lett. 31, 1041 (1973).

20) I. B. Bernstein, and S. K. Trehan, Nucl. Fusion 1, 3 (1960).

21) J. J. Rasmussen and H. L. Pécseli, Phys. Lett. 55A, 85 (1975).

22) V. P. Popovitch and I. F. Kharchenko, Proc. 12th International Conference on Phenomena in Ionized Gases, Findhoven, Aug. 18-22 1975, (North Holland, 1975) p. 280 . 


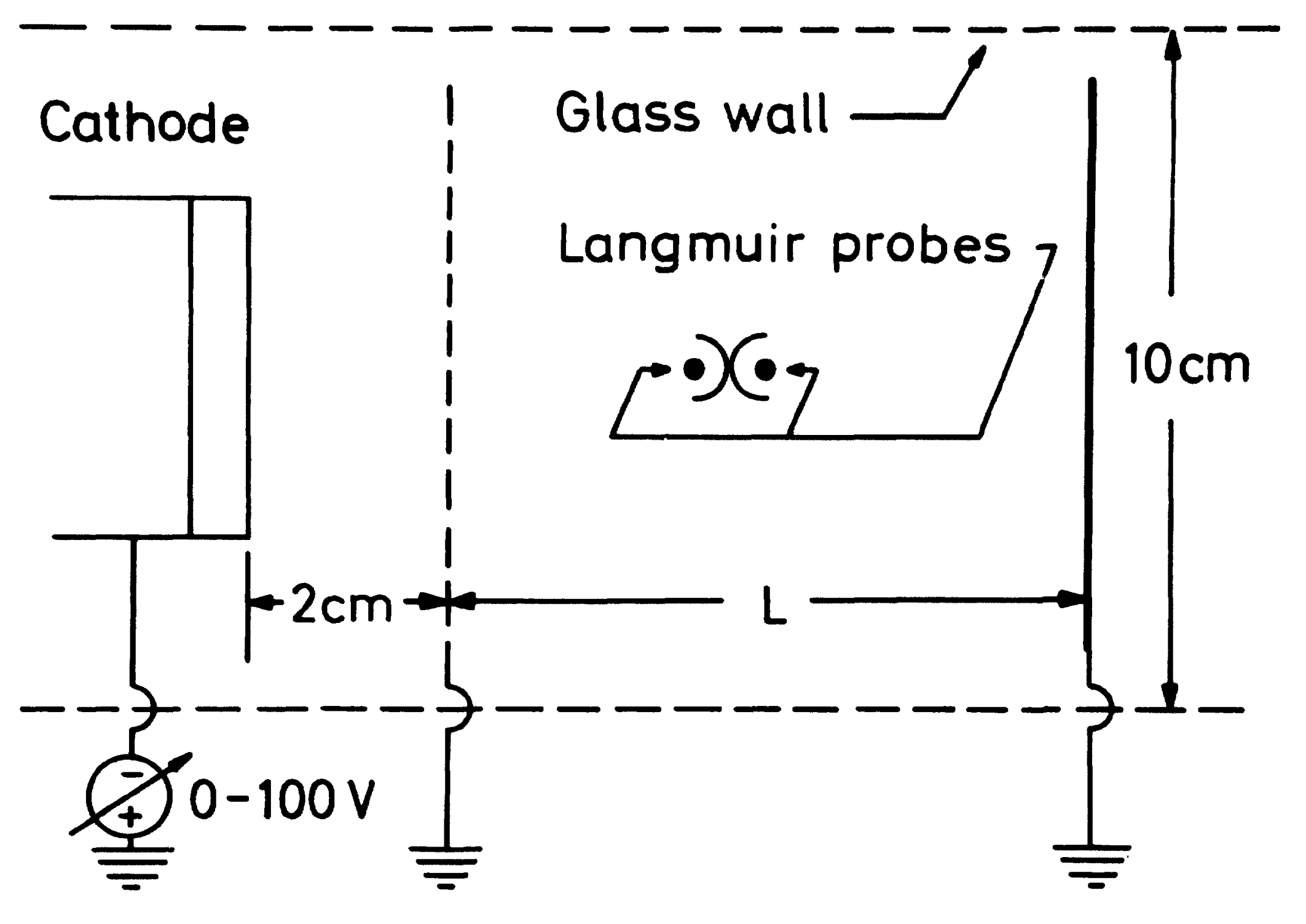

Fig. 1. Schematic diagram of experimental set up. 


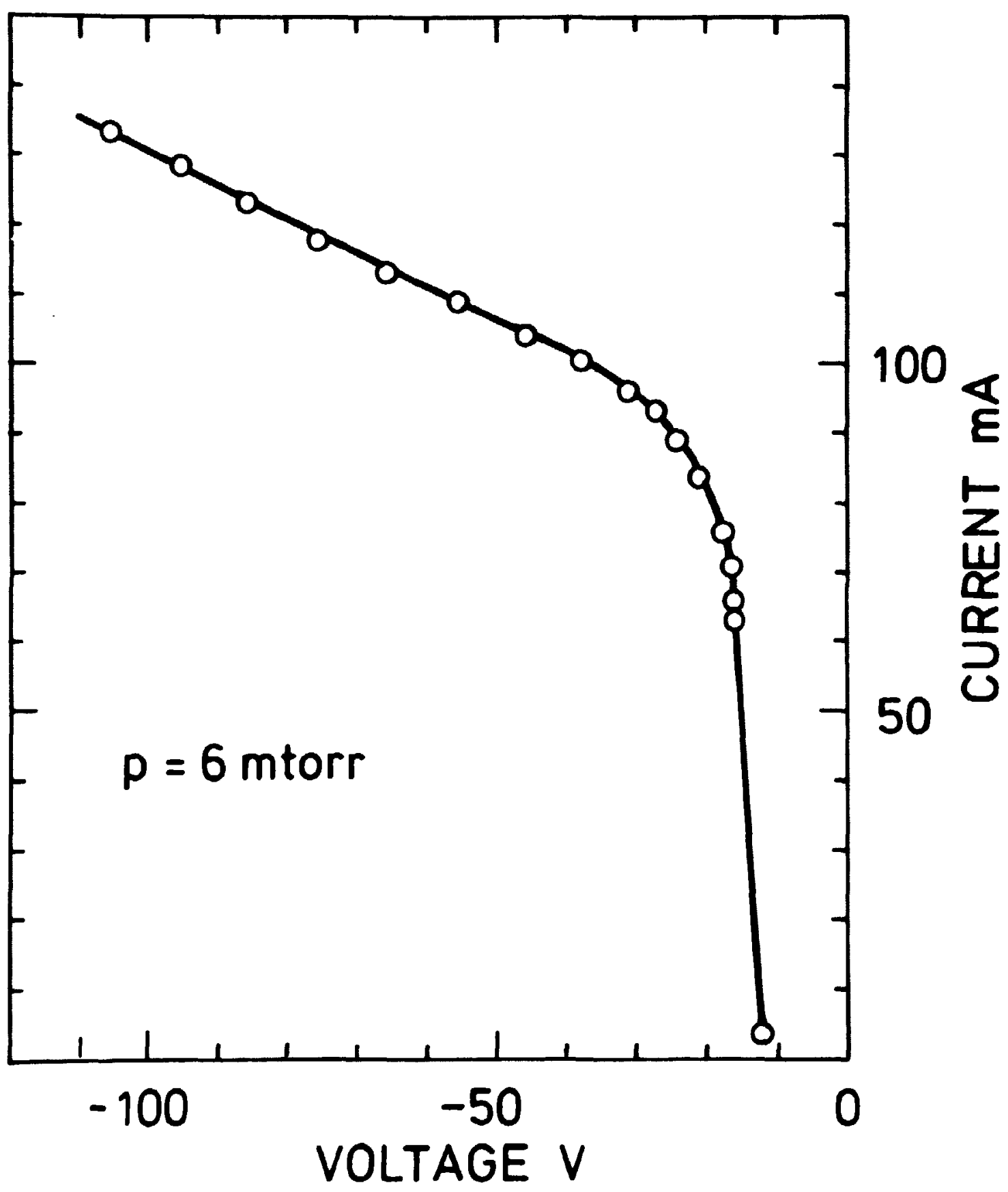

Fig. 2. Current-voltage characteristics of the discharge. Length of plasma column $L=25 \mathrm{~cm}$. The ionization potential for argon is $15.76 \mathrm{eV}$. 


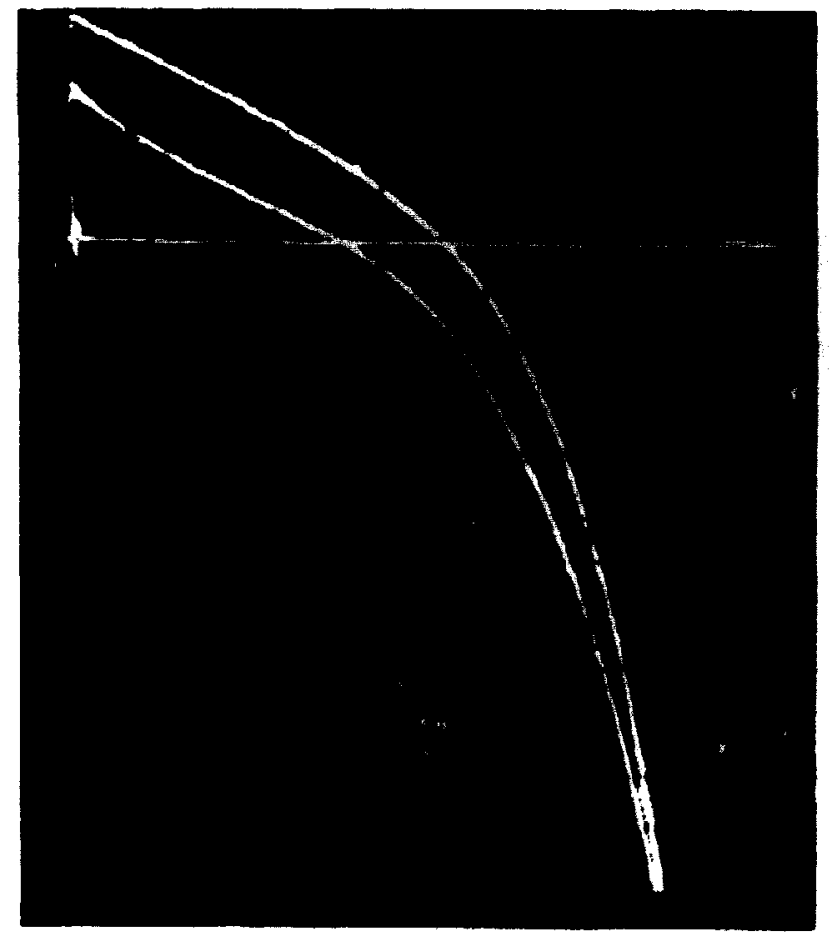

Fig. 3. Probe characteristics. Upper trace from vrofe facino awy from the gria (Prote 2), lower trace frem probe

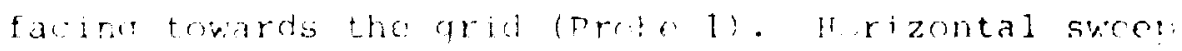

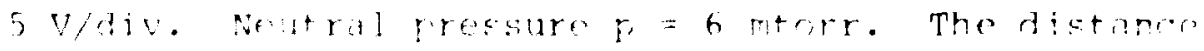

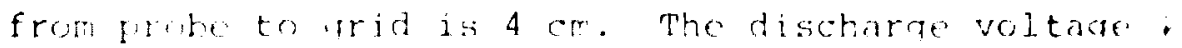
$-25 v$. 


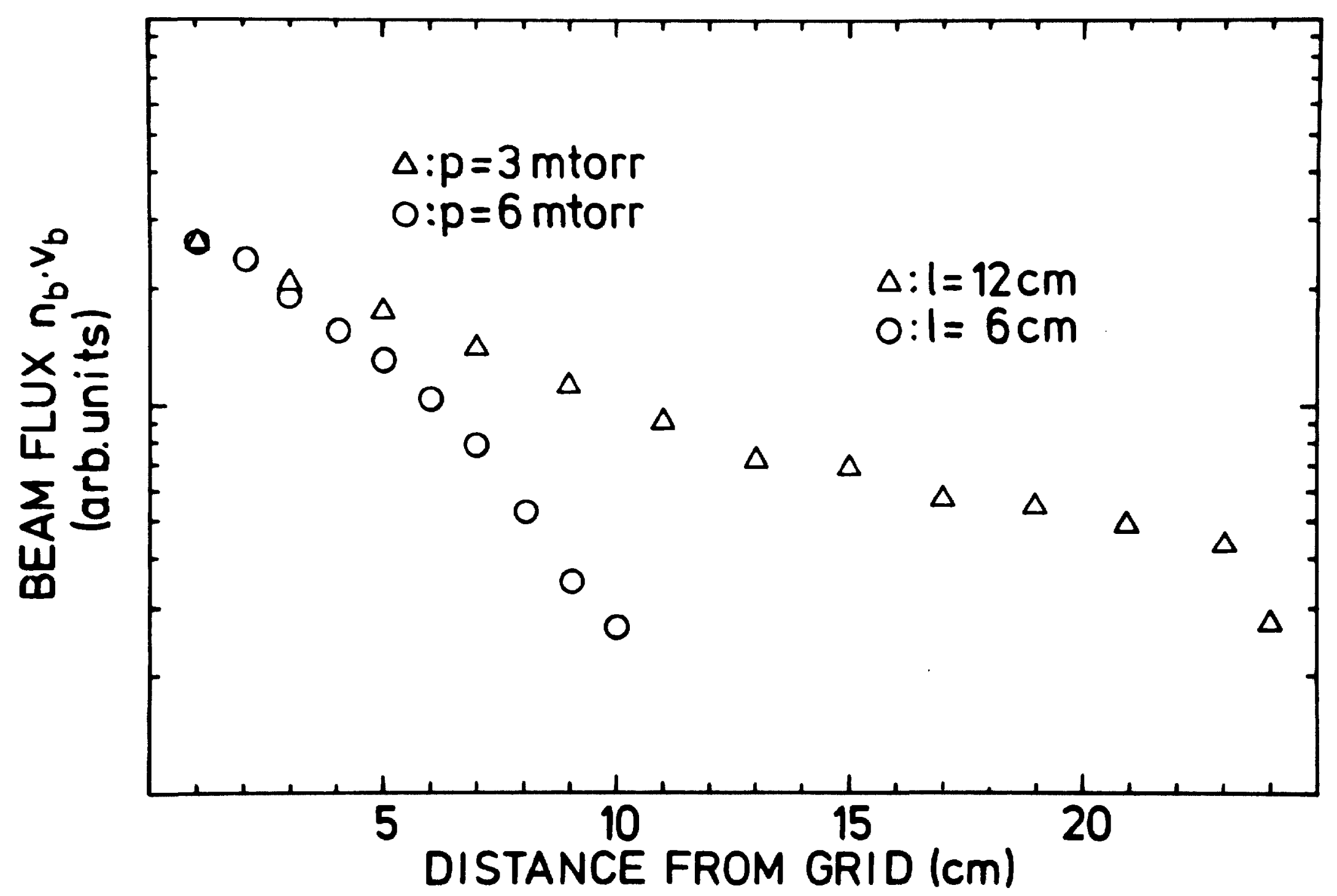

Fig. 4. a) Axial variation of beam fluxes $n_{b} \cdot v_{b}$ measured on probe 1. Discharge voltage $\Delta: \phi=-25 \mathrm{~V}, 0: \phi=-29 \mathrm{~V}$, $l$ is the mean free path for collisions. 
BACKGROUND DENSITY $n$

(arb. units)

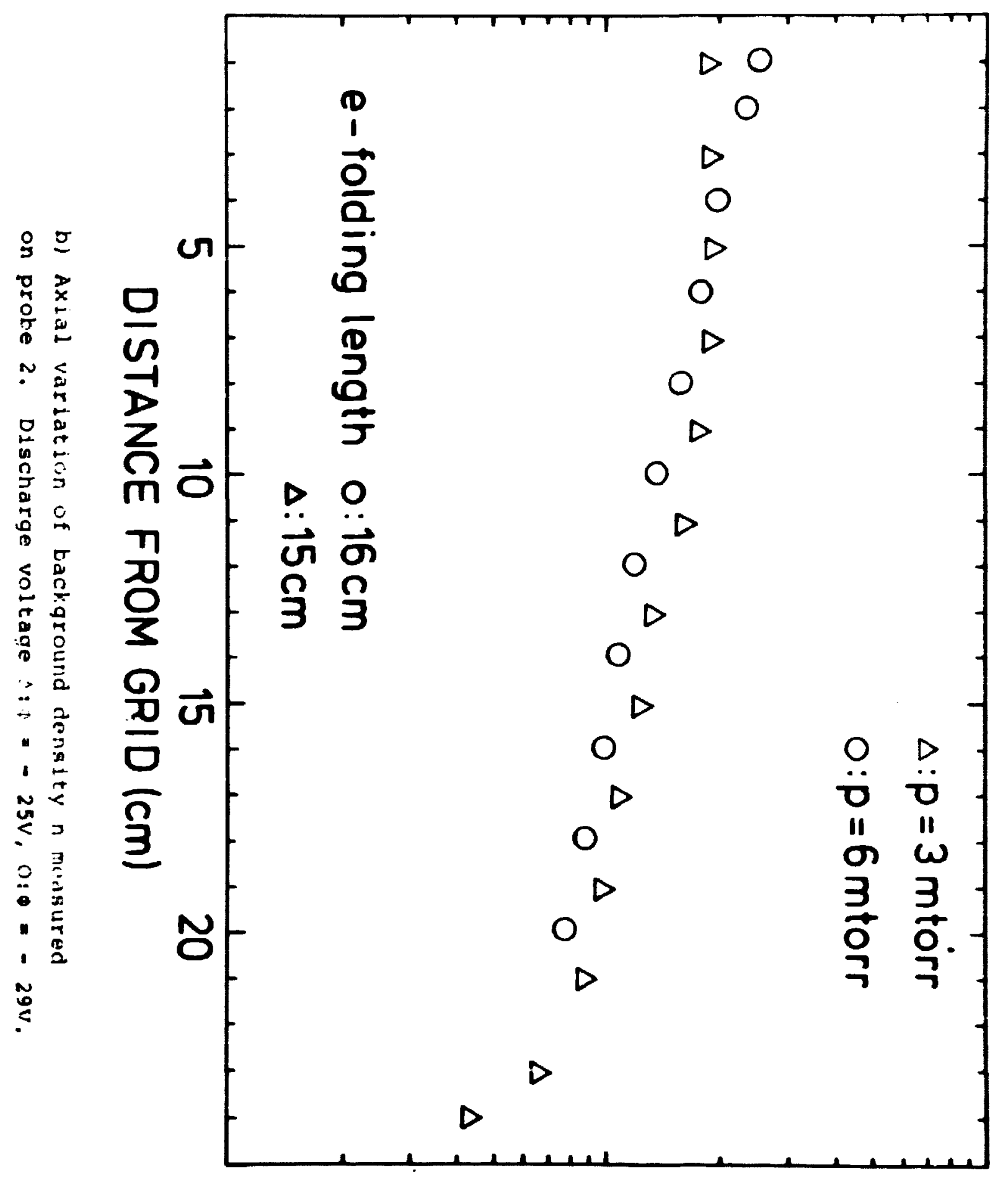



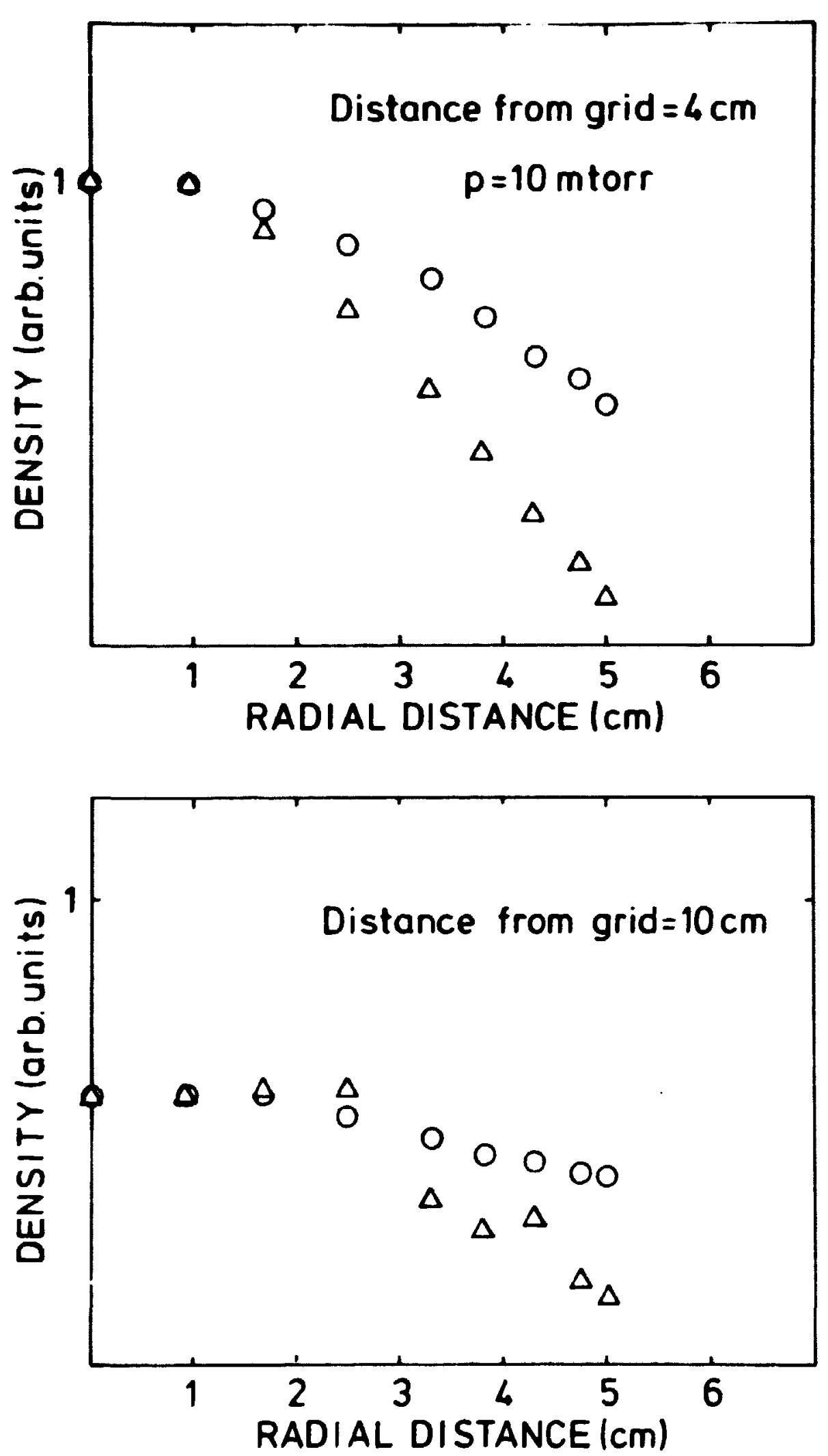

Fig. 5. Radial variation of background density $n$ wi. normal : beam flux $n_{b} v_{b} / n$ measured on proke 2 and $p r i r n 1$, respectively. Discharge voltage $\phi=-50 \mathrm{~V}$. 


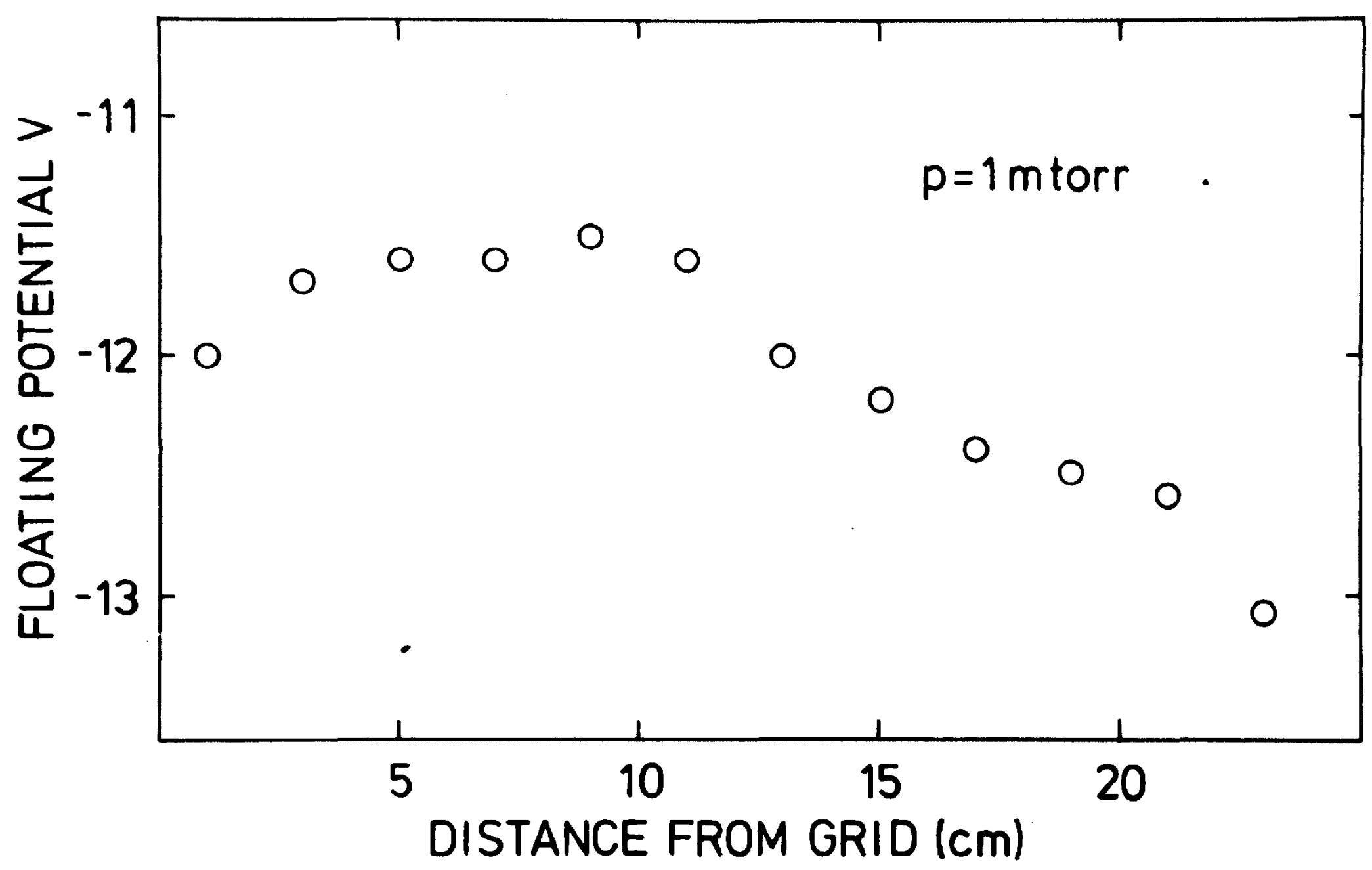

Fig. 6. Axial variation of the floating potential measured on probe 2. Discharge voltage $\Phi=-50 \mathrm{v}$. 


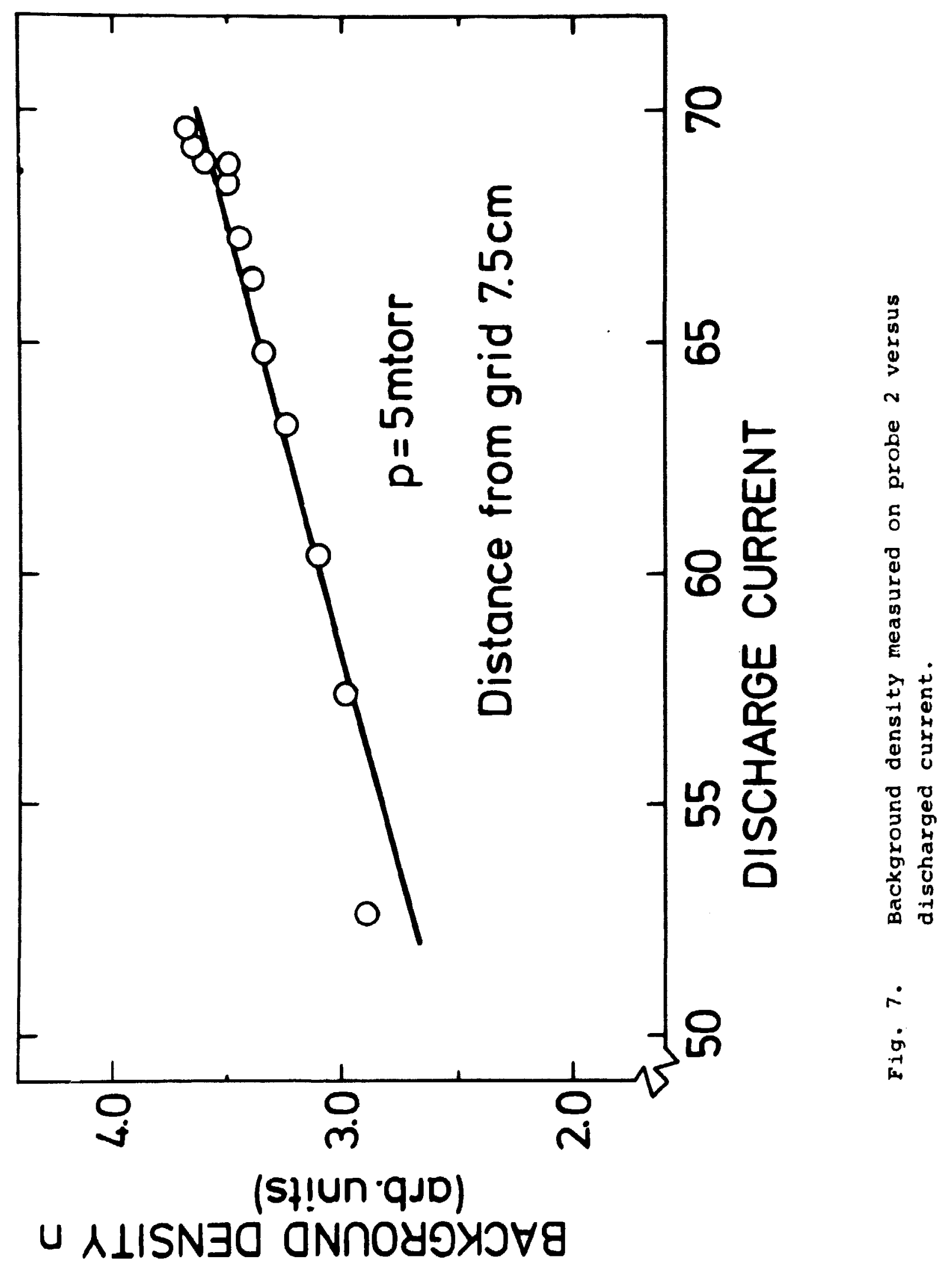



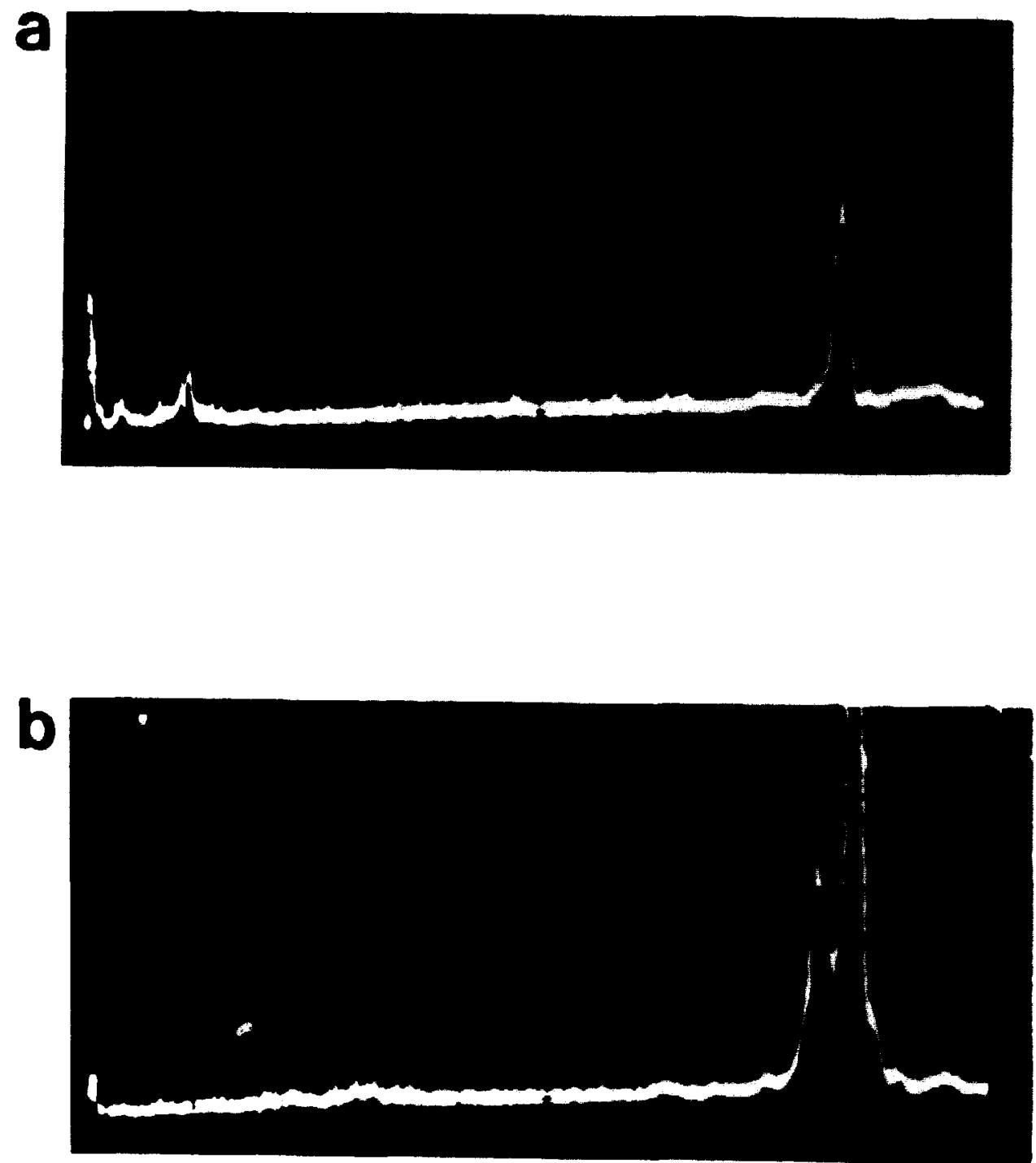

Fig. 8. Typical spectra, horizontal sveep O - $1250 \mathrm{MHz}$. a) Measured on the Langmuir prohe in the plasma. Neutral pressure $p=18$ mtorr. Discharge voltage $=-80 \forall$. Length of plasma column, $\mathrm{I},=25 \mathrm{~cm}$. b) Measured on the antenna outside the plasma, same condition as a). 

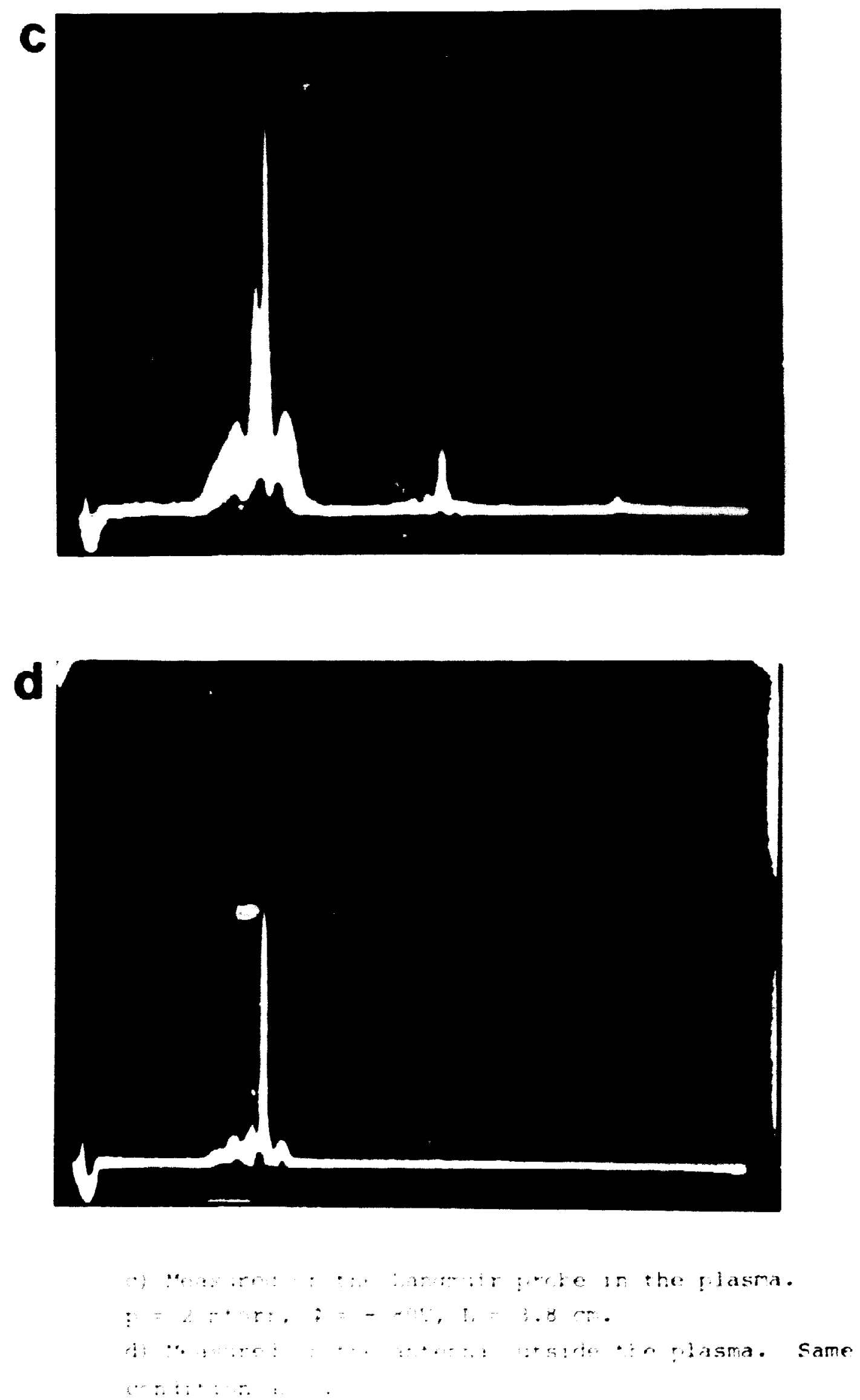

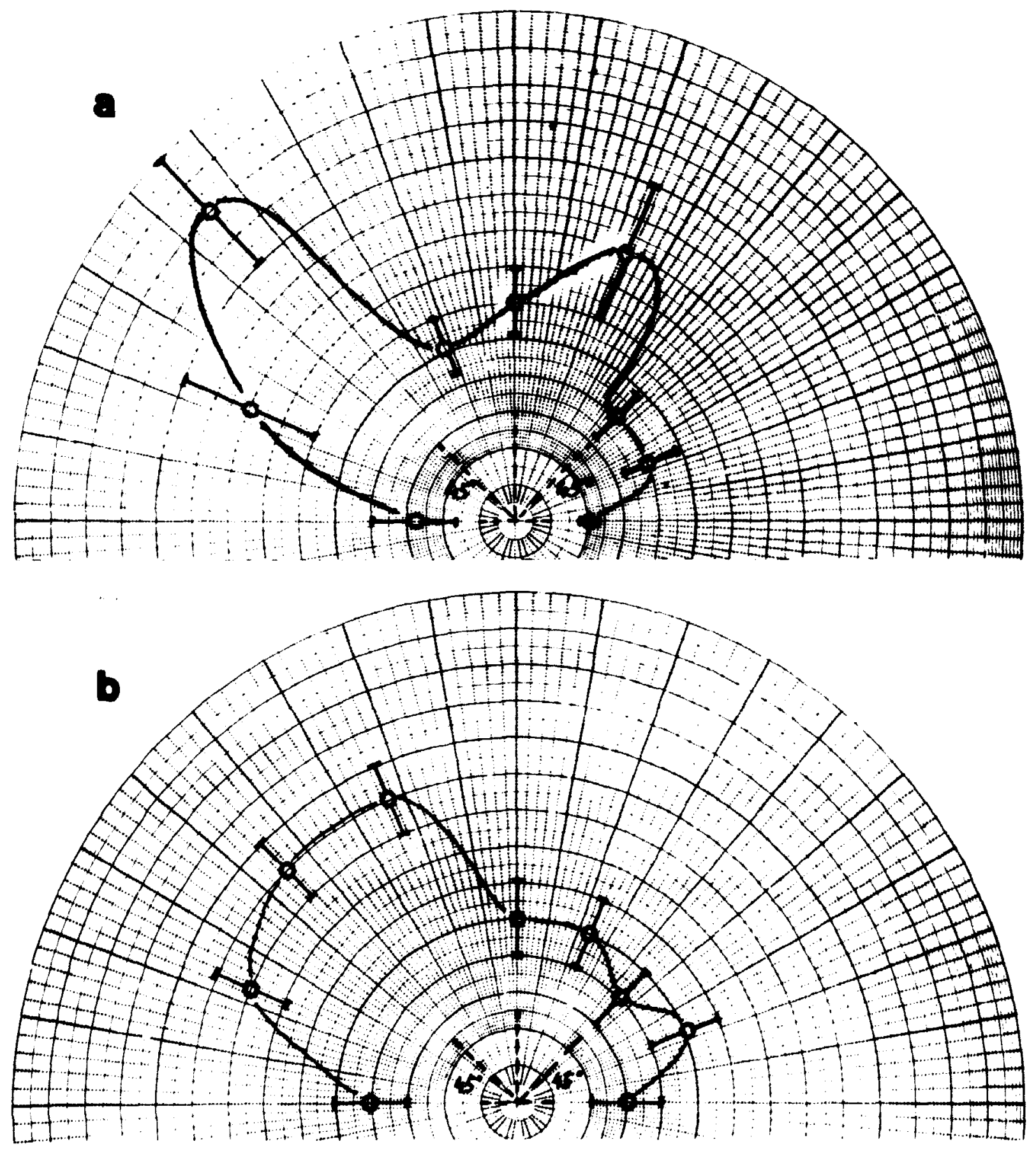

Fig. 9. Radiation patterns

a) Neutral pressure $p=2$ mtorr. Discharge voltage $\phi=$

$-75 \mathrm{v}$. Length of plasma column, $\mathrm{L}=3.8 \mathrm{~cm}$.

b) $\mathrm{p}=2$ mtorr, $\phi=-75 \mathrm{~V}, \mathrm{~L}=5 \mathrm{~cm}$. 


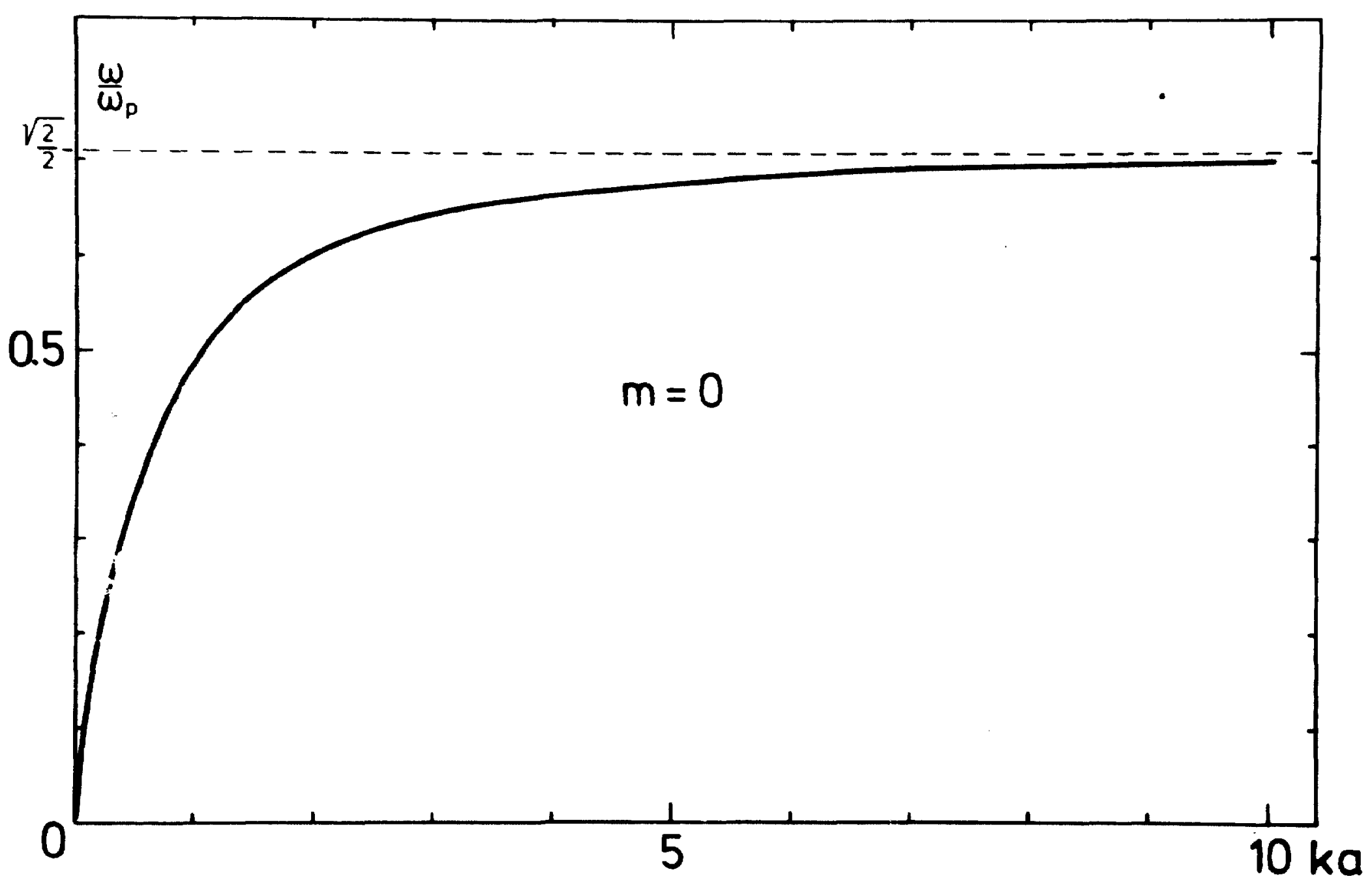

Fig. 10. The dispersion relation: $u^{2}={ }_{p}^{2} I_{m}^{\prime}(k a) k_{m}(k a) k a$.

$n=5 \mathrm{mu}$ is. 


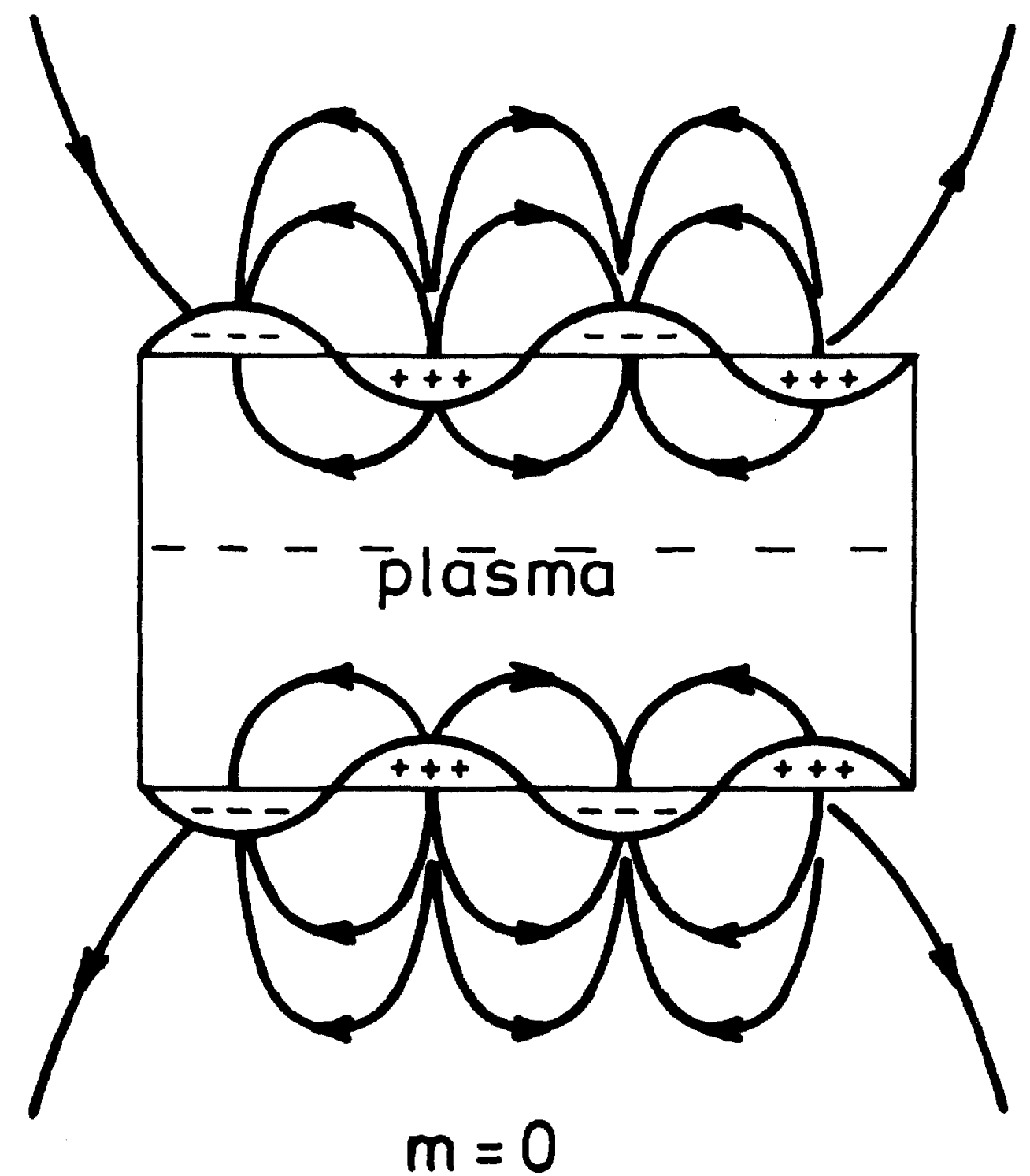

Fig. 11. Distribution of the electric field lines outside the plasma. 


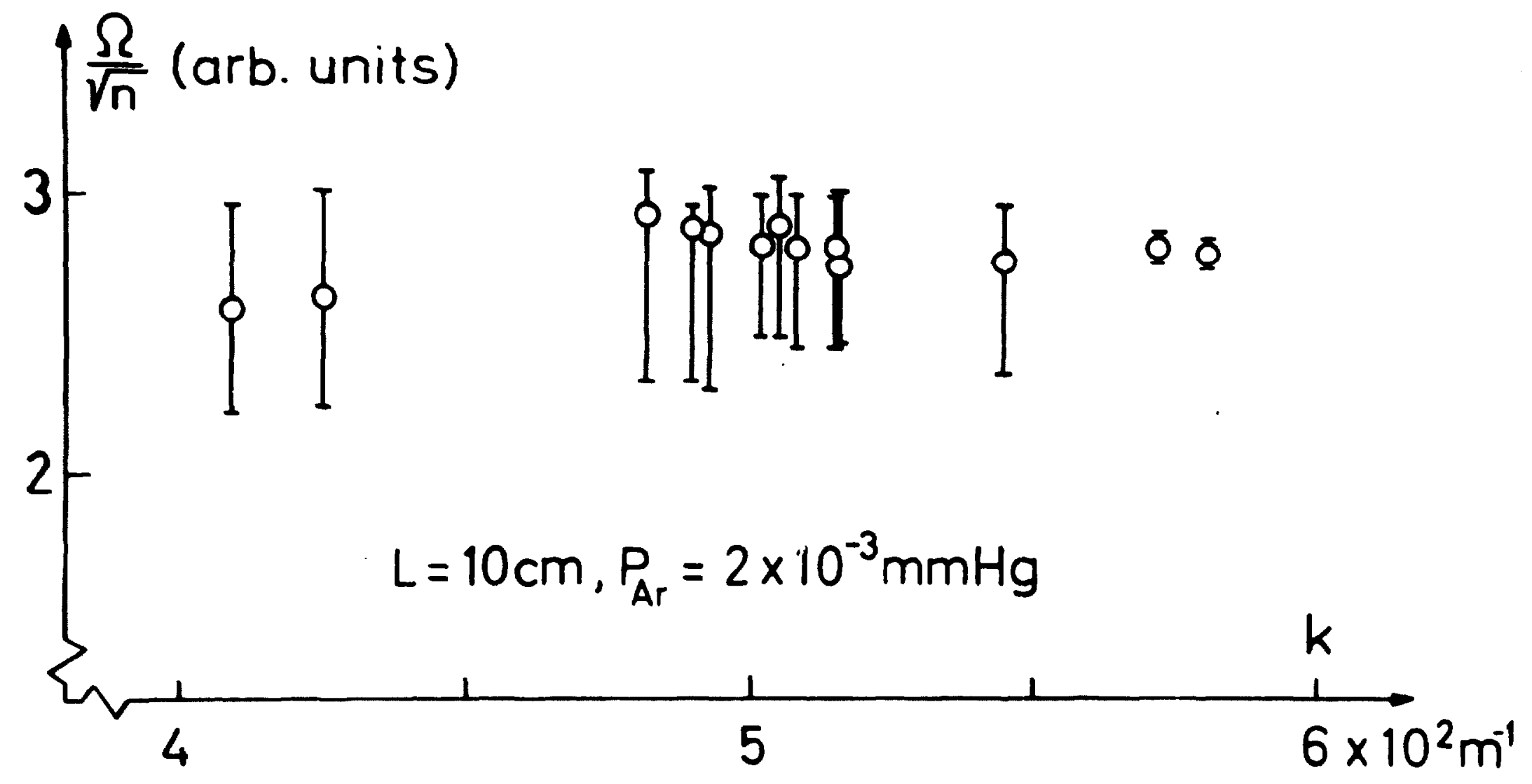

Fig. 12. Normalized observed frequency $/ \mathrm{rn}^{\mathrm{v}}$ versus $k=\mathrm{V}_{\mathrm{r}}$. The error bars indicate the band-width of the oscillations while the circle shows the frequency with maximum amplitude. 


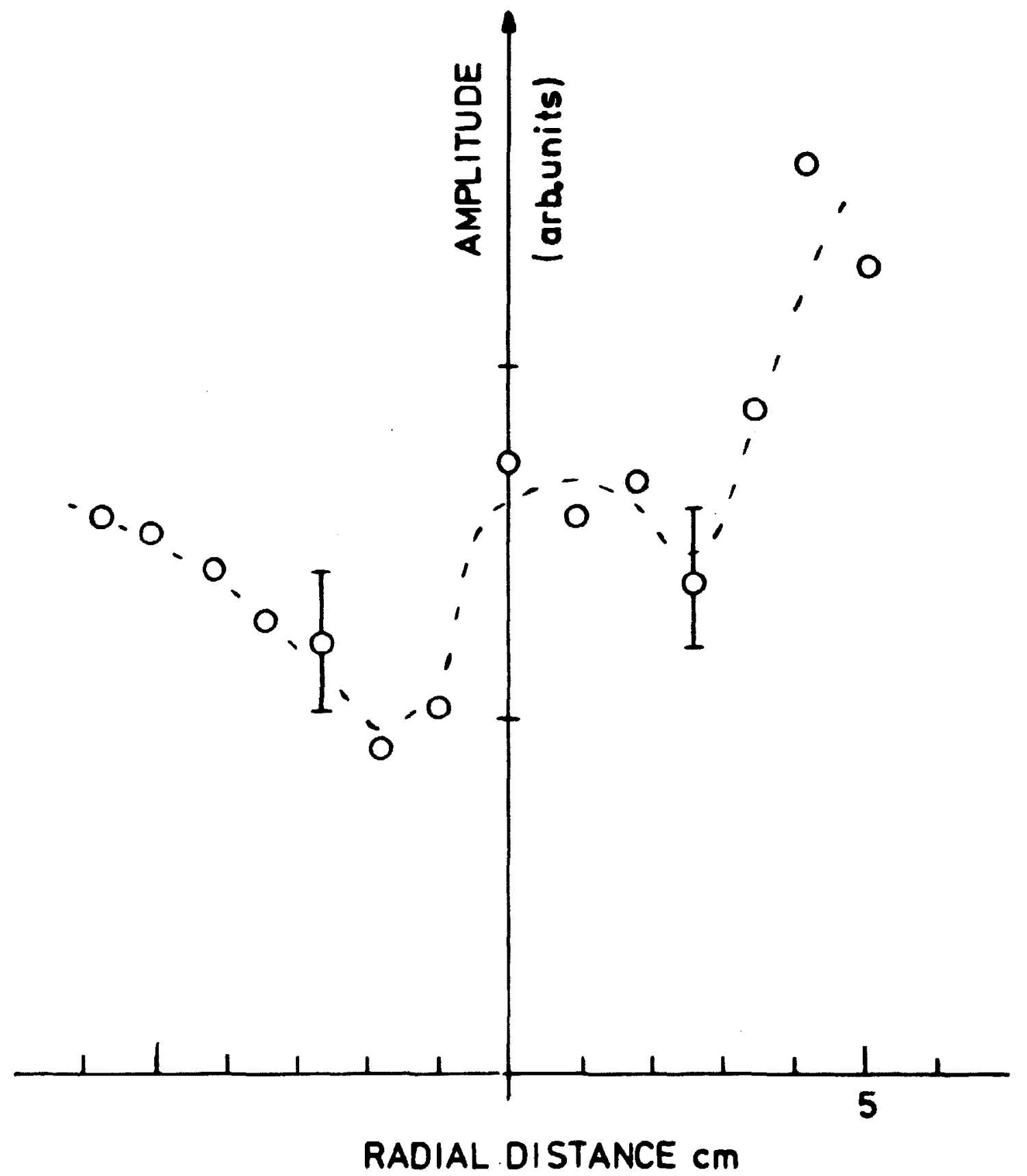

Fig. 13. Radial variation of oscillations. Neutral pressure $P=2.5$ mtorr. Discharge voltage $\phi=-81 \mathrm{~V}$. 


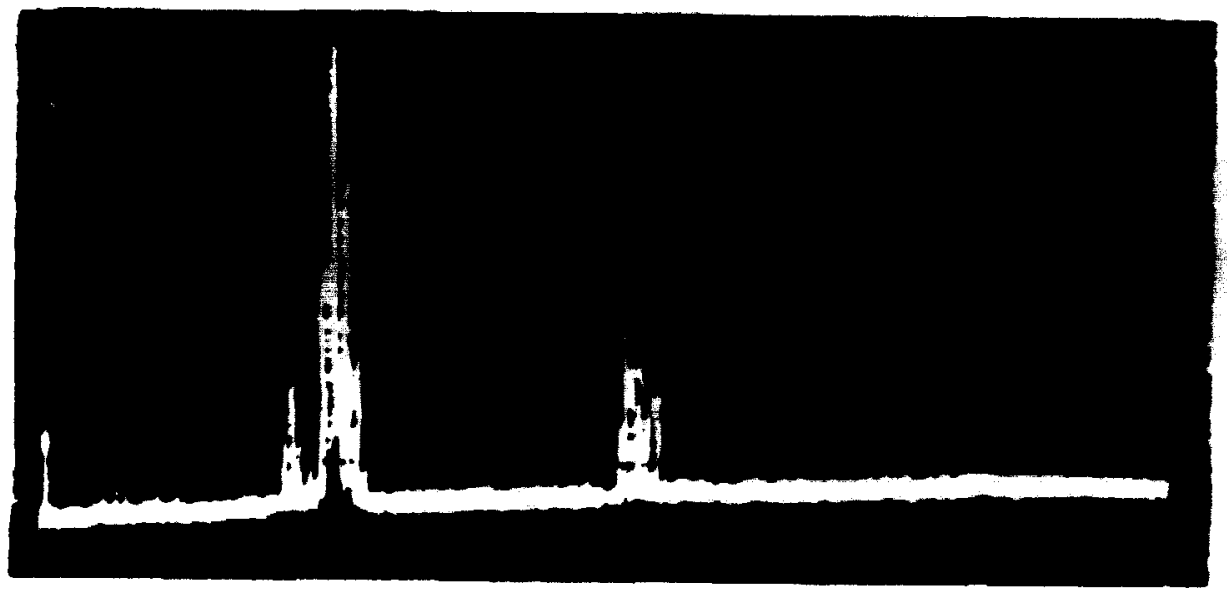

Fig. 14. Spectrum showing a case where second harmonics are generated. 